恼

JOURNAL OF ETHNOBIOLOGY

AND ETHNOMEDICINE

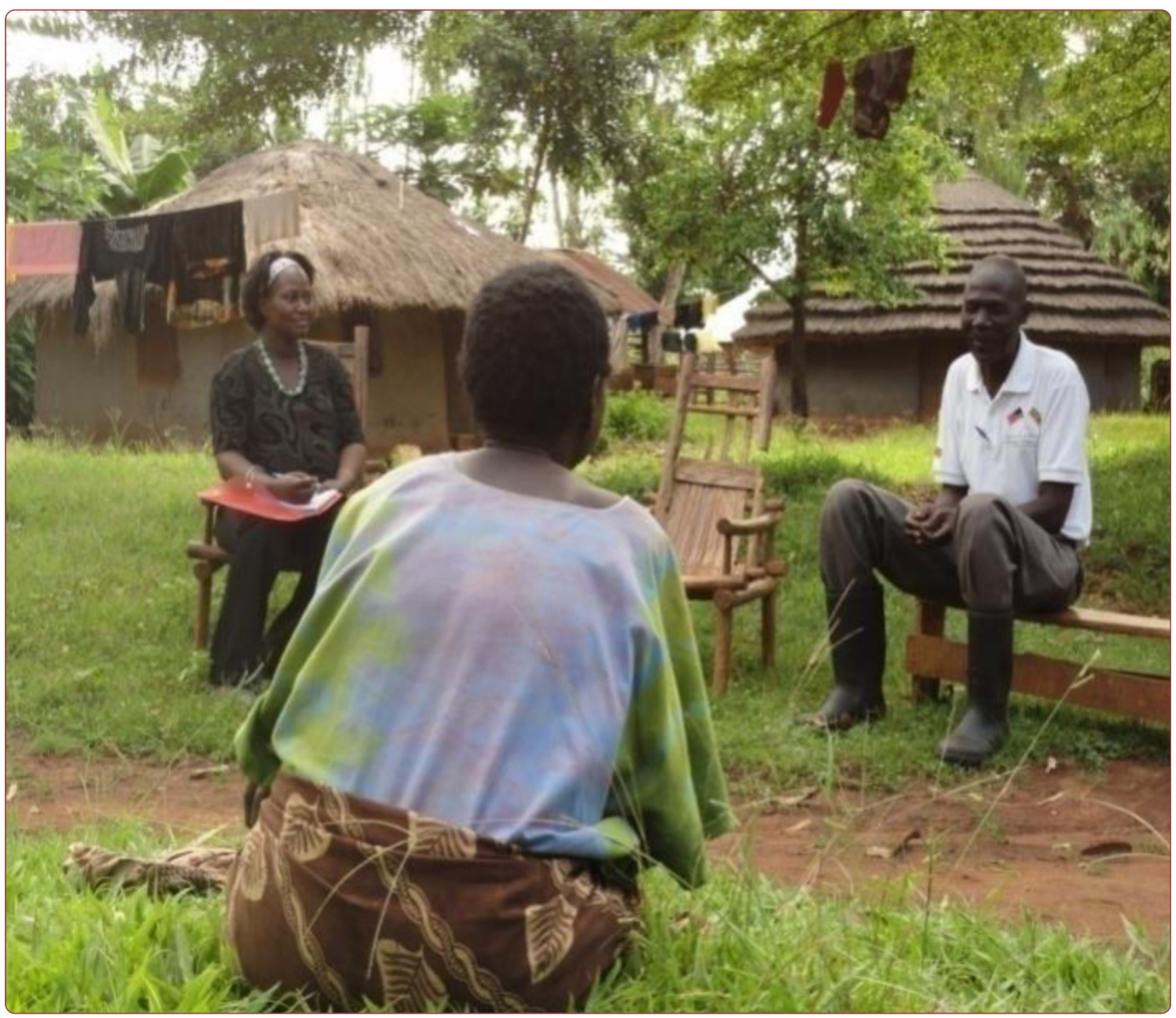

\title{
Ethnopharmacological practices by livestock farmers in Uganda: Survey experiences from Mpigi and Gulu districts
}

Nabukenya et al. 


\title{
Ethnopharmacological practices by livestock farmers in Uganda: Survey experiences from Mpigi and Gulu districts
}

Immaculate Nabukenya ${ }^{1,3^{*}}$, Chris Rubaire-Akiiki ${ }^{1}$, Deogracious Olila ${ }^{1}$, Kokas Ikwap ${ }^{1}$ and Johan Höglund²

\begin{abstract}
Background: There is continued reliance on conventional veterinary drugs including anthelmintics, to some of which resistance has developed. Loss of indigenous technical knowledge (ITK) from societies affects the opportunities for utilization of ethnopharmacological practices unless properly documented. This study was conducted to identify common traditional practices using medicinal plants against helminthosis and other livestock diseases in Mpigi and Gulu districts of Uganda.
\end{abstract}

Methods: Seven focus group discussions with ten farmers per group plus 18 key informant interviews were held in each district from August to November 2011. Ranking was used to quantify disease burdens and to identify priority livestock and breeds. Samples of each plant were submitted to Makerere University herbarium for identification and documentation. The local name, relative availability and International Union for Conservation of Nature (IUCN) status were recorded.

Results: Seventy six farmers in Mpigi and 74 in Gulu were interviewed. Theileriosis and helminthosis were the most common disease conditions in cattle and goats, respectively. Forty plant species within 34 genera from 22 botanical families were identified, with 20 of these used against helminthosis. Other plants treated wounds and ecto-parasites, theileriosis, retained placenta and bovine ephemeral fever. Non-plant practices (7) and plants cited were used in combination depending on availability. Males older than 40 years had most ethnopharmacological knowledge. Most plants $(75 \%, n=40)$ were common, but 10 were rare. IUCN status was not evaluated for $95 \%$ of these plants. Conventional and traditional drug use in Gulu and Mpigi districts was different $\left(x^{2}=24 ; p<0.001\right)$. The scientific, English, Luganda and Acholi names of all plants and their availability within the communities are documented herein.

Conclusion: This is the first detailed livestock-related ethnopharmacological study in Gulu district. Farmers in Uganda are still using a variety of practices to treat livestock ailments. Scientific validation and evaluation of conservation status are urgently needed to ensure future availability and knowledge about these plant resources.

Keywords: Ethnopharmacological practices, Herbarium, ITK, Plants, Medicine, Helminthosis, Conservation

\footnotetext{
* Correspondence: lennfaith@yahoo.com

'College of Veterinary Medicine, Animal Resources and Biosecurity, Makerere

University, P. O. Box 7062, Kampala, Uganda

${ }^{3}$ Department of Biosecurity, Ecosystems and Veterinary Public Health, P. O.

Box 7062, Kampala, Uganda

Full list of author information is available at the end of the article
}

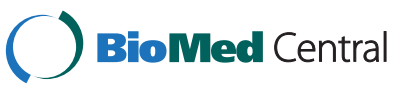

(c) 2014 Nabukenya et al.; licensee BioMed Central Ltd. This is an Open Access article distributed under the terms of the Creative Commons Attribution License (http://creativecommons.org/licenses/by/2.0), which permits unrestricted use, distribution, and reproduction in any medium, provided the original work is properly cited. The Creative Commons Public Domain Dedication waiver (http://creativecommons.org/publicdomain/zero/1.0/) applies to the data made available in this article, unless otherwise stated. 


\section{Background}

Cattle and goats are the main livestock ruminants kept in rural communities in tropical Africa [1]. In Uganda, more than $80 \%$ of the rural population relies on agricultural production, with a varied focus on livestock and crop agriculture depending on the agro-ecological zone [2]. Livestock diseases greatly affect animal welfare, health and productivity, and lead to high treatment costs, losses through reduced growth, unchecked morbidity and mortality $[1,3,4]$. Helminthosis is among the most debilitating livestock conditions, costing farmers millions of Ugandan shillings through lost production and control efforts $[2,5,6]$. Notably, gastrointestinal nematodes cause poor weight gains, reduced production, severe weight loss or even direct mortality, especially in small ruminants in resource-poor farming communities [1]. Although many alternative control strategies for helminthosis, such as pasture management [7-9], use of nematophagous fungi $[10,11]$, nutritional supplementation with beneficial forages [12-15], and targeted anthelmintic treatment with the FAMACHA ${ }^{\odot}$ system, have been put forward [16,17], frequent use of anthelmintics dominates [4], to which helminths have become resistant [18-20].

The World Health Organization estimates that about $80 \%$ of the population in developing countries depends on traditional medicine for their primary health care needs [21]. Most of the rural small-holder farmers cannot afford the cost of modern drugs [22], and therefore resort to Indigenous Technical Knowledge (ITK). ITK refers to localized, structured, traditional application of knowledge generated from continuous experimentation and observation of a given phenomenon of interest [23]. In this study, it refers to the use of ethnopharmacological practices, which over a long time have been used to treat diseases and ailments in a given geographical location. The study of ITK is encouraged in Uganda and many African countries because ethnoveterinary practices can supplement modern drugs, and since they are cheaper [24] they are often practiced by farmers $[5,22,25]$. In addition, drug discovery efforts have been refreshed with the aim of addressing the current resistance problems against the most frequently used anthelmintics [26].

There are a number of studies in Uganda which have documented livestock species-specific ethnopharmacological practices in different parts of the country [25,27-33]. However, due to limited scope, variation of ITK by culture and changing livestock production practices, diversified knowledge from other regions is needed. Before 1986, the Acholi people in Gulu produced a large proportion of cattle and goats, but the district was ravaged by war for more than 20 years. With peace returning, Gulu farmers are currently restocking the land. In contrast, Mpigi district is part of what is known as the cattle corridor, where livestock production is highest in Uganda. Being near the capital city, it is easy to access modern veterinary drugs. With traditional knowledge being replaced by continued use of conventional veterinary drugs, the utilization of indigenous practices is expected to decrease unless properly documented [1]. To document such indigenous knowledge, this study was undertaken to identify the most common practices using plants to treat livestock diseases in Mpigi and Gulu districts of Uganda.

\section{Materials and methods}

\section{Study design, area and sampling}

A cross-sectional survey was conducted from August to November 2011 in two districts: Mpigi and Gulu. Mpigi district is located in the central region between $00^{\circ} 13^{\prime}$ $48^{\prime \prime}$ North and $32^{\circ} 19^{\prime} 48^{\prime \prime}$ East coordinates. The headquarters are located $37 \mathrm{~km}$ west of the capital Kampala, and it is part of the intensive livestock/cattle farming corridor in Uganda (Figure 1). Gulu district is located about $340 \mathrm{~km}$ north of Kampala, between $02^{\circ} 45^{\prime}$ North and $32^{\circ} 00^{\prime}$ East coordinates, with a population that relies on subsistence agriculture [34]. Multistage sampling was used to select the study sites. In each district, two subcounties were selected. Subsequently, 3 villages in each sub-county were purposively selected [27] with the help of extension staff, making a total of 6 villages in each district.

\section{Data collection}

An assessment of the different ethnopharmacological practices and beliefs among cattle and goat farmers was completed using standard participatory rural appraisal (PRA) techniques [35]. These included focus group discussions (FGDs) with local farmers, key informant interviews (KIIs), scoring and ranking, as well as problem mapping. Visits to collect plant materials and botanical identification were carried out.

\section{Group discussions}

In Mpigi, seven FGDs were conducted in Kammengo, Bwanya, Mpigi Town Council, Bulamu, Konkoma and Jeza villages. In Gulu, seven FGDs were held in Bobi, Unyama, Awach, Purong, Ombachi and Custom Central villages. These villages were selected because they represented the peri-urban and rural agricultural production systems. Seventy six and 74 farmers were interviewed in Mpigi and Gulu, respectively, with an average of 10 people (both male and female) per group. Participants were livestock farmers owning at least five ruminants and with more than five years of livestock farming experience. A standard interview guide was used to ensure uniformity of the data collected during the FGDs, after the participants had signed a consent form. The discussions were taped and kept in the local languages (Luganda and Acholi) but later translated for the researchers. 


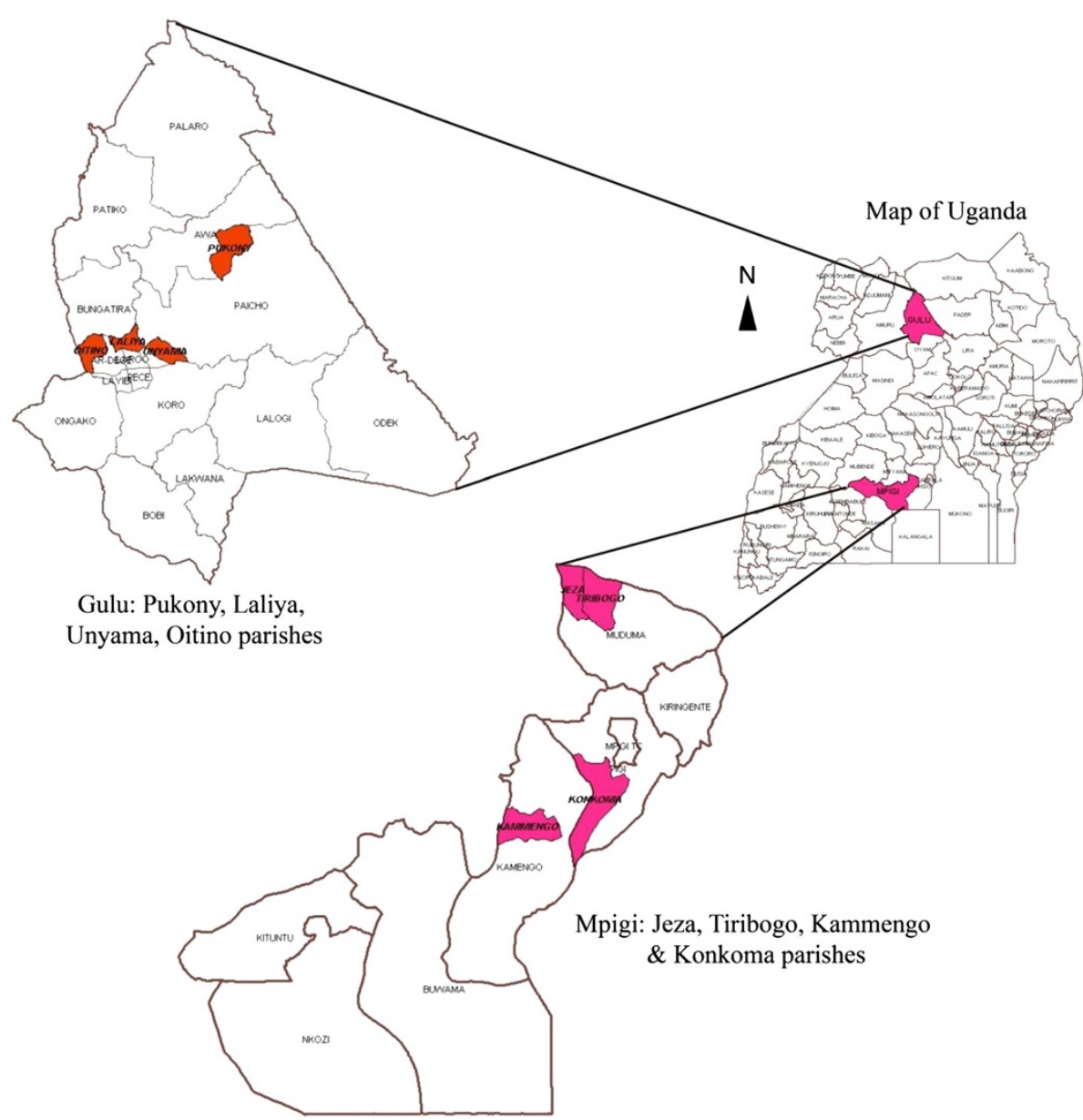

Figure 1 Map of Uganda showing the two study districts, Gulu and Mpigi.

\section{Interviews}

In each district, nine KIIs were held, four with veterinary extension workers and five with community ITK persons, using a semi-structured interview guide. Both in the KIIs and FGDs information was gathered on priority livestock and prevailing diseases including helminthosis. Perceived causes, disease diagnosis, treatment methods, plant sources, herbal preparations and dosages were documented. The conservation efforts and challenges encountered while using herbal medicine were also documented.

\section{Matrix scoring and ranking}

To understand how diseases were diagnosed and treated, participants were involved in practical scoring and ranking of diseases and their treatment. The participants were provided with 100 bean seeds each, which they used to indicate differences in availability of the plant species or varieties mentioned, and changes in burden of disease with livestock species and general priorities. This helped to quantify some items in the checklists and achieve agreement on a given variable within the group.

\section{Plant identification}

A local name was attached to each plant, and information on its relative availability was recorded [31]. Reference samples of each plant were submitted to Makerere University herbarium for identification by a botanist, and voucher specimens were preserved. Review of each plant's conservation status data was done in reference International Union for Conservation of Nature (IUCN) red list (www.iucnredlist.org) and Convention on International Trade in Endangered Species (CITES) classification (http://www.cites.org).

\section{Data management and analysis}

The data were transcribed and entered into Microsoft Excel 3.0. During analysis, data were summarized into major themes by content analysis [35]. Descriptive statistics were obtained for quantifiable data in Statistical 
Package for Social Scientists (SPSS version 15.0). The scientific family and species names of the plants were obtained after botanical identification.

\section{Results}

\section{Socio-demographics of respondents}

A total of 150 participants were interviewed in seven FGDs conducted in each district. Of these, 87 (58\%) were female and $63(42 \%)$ male. On average, the farmers interviewed were $36 \pm 9.8$ years old (mean \pm standard deviation). Whereas 15 (10\%) participants had no formal education, 83 (55\%) had primary and 62 (35\%) secondary education level. The average number of goats was $8.6 \pm 3.9$ with a minimum of 2 and maximum of 56 goats. The average cattle number was $3.2 \pm 2.9$ with a maximum of 49 . Twenty percent $(n=$ 150) of the farmers had only goats. The average household land size was $2.5 \pm 2.9$ acres (range 1-30) in Mpigi and $6.5 \pm 3.5$ acres (range 3-50) in Gulu district.

\section{Major livestock species kept}

FGDs in Mpigi revealed that the priority livestock was local breeds of cattle followed by chicken (Table 1). Most $(>70 \%)$ of the pigs, goats and sheep were local breeds, with few crosses and exotic breeds. Species ranked highly in Gulu were goats followed by cattle. Most of the chicken, cattle and goats were local breeds, whereas pigs were exclusively exotic or cross breeds (Table 1 ).

\section{Prevalent livestock diseases and conditions}

Theileriosis or East coast fever (ECF), known as "Amakebe" in Luganda and "Oding ding" in Acholi, was ranked by the farmers as the most common cattle disease in both districts (Table 2). Helminthosis was a commonly encountered condition in all priority species. It was ranked number one in goats and sheep, second in pigs, but was less important in chicken (Table 2). Descriptions of the most common diseases and conditions during interviews were usually accurate. Notably, ECF was not well understood, with farmers claiming that it mostly affects young cattle whose lymph nodes swell when they consume a lot of milk. Helminthosis was well described, including clinical signs such as emaciation and weight loss, rough hair coat, reduced appetite, "pot belly" and detection of worms or segments in faecal matter. Apart from farmers with exotic livestock and chicken breeds, $80 \%$ of the local farmers did not regularly treat against helminthosis. However, they treated affected animals, especially when some had diarrhoea or died.

\section{Treatment of animal diseases}

Treatment depended on availability of funds to procure the conventional drugs (49\%), availability of veterinary services (22\%), knowledge of alternatives such as ITK (15\%), cost attached to the animal (10\%) and seriousness of the condition (6\%). Owing to low availability of service providers, $55 \%$ of the farmers treated their own animals. The majority (60\%) of the farmers used the conventional drugs to treat their animals especially cattle, pigs and chicken (Figure 2). Generally during discussions, participants aged below 40 years showed less knowledge about alternative treatment options and ITK. This was more pronounced in Gulu than in Mpigi district where the different plant names and their uses were discussed by farmers older than 40 years. Relatedly, $25 \%$ of the farmers with knowledge relied entirely on the alternative herbal preparations to treat animal diseases (Figure 2), as demonstrated in this excerpt:

\section{"We used the plants before, but we learnt over time that when you want maximum returns or when your animals are crosses and exotic breeds, the choice is to use veterinary drugs, thus we changed" - Female FGD Respondent, Mpigi district.}

The reasons for low level of use among the knowledgeable farmers were: increased trust in conventional drugs $(60 \%)$, scarcity of the plant resources (30\%) and lower demand from other farmers (10\%). There was a significant

Table 1 Priority livestock species and common breeds in Mpigi and Gulu districts

\begin{tabular}{|c|c|c|c|c|c|c|c|c|}
\hline \multirow[b]{2}{*}{ District } & \multirow[b]{2}{*}{ Livestock species } & \multirow[b]{2}{*}{ Priority \% } & \multicolumn{3}{|l|}{ Breeds } & \multicolumn{3}{|c|}{ Management system } \\
\hline & & & Local \% & Crosses $\%$ & Exotic \% & Free range $\%$ & Semi-intensive* \% & Intensive \% \\
\hline \multirow[t]{4}{*}{ Mpigi } & Cattle & 35 & 47 & 48 & 5 & 15 & 80 & 5 \\
\hline & Chicken & 30 & 47 & 3 & 50 & 50 & 0 & 50 \\
\hline & Pigs & 25 & 80 & 17 & 3 & 0 & 80 & 20 \\
\hline & Goats \& sheep & 10 & 85 & 13 & 2 & 5 & 90 & 5 \\
\hline \multirow[t]{4}{*}{ Gulu } & Goats** & 50 & 80 & 15 & 5 & 20 & 78 & 2 \\
\hline & Cattle & 30 & 80 & 15 & 5 & 90 & 5 & 5 \\
\hline & Pigs & 10 & 5 & 50 & 45 & 10 & 20 & 70 \\
\hline & Chicken & 10 & 90 & 2 & 3 & 90 & 0 & 10 \\
\hline
\end{tabular}

*Semi-intensive management method is tethering (tying animals with a rope at a different fixed grazing/feeding area each day).

**Sheep are much less common; preference for goats overrides. 
Table 2 Most prevalent livestock and chicken diseases in Mpigi and Gulu districts

\begin{tabular}{|c|c|c|c|c|c|}
\hline $\begin{array}{l}\text { Animal species } \\
\text { affected }\end{array}$ & Disease name & Local name - Luganda & Local name - Acholi & $\begin{array}{l}\text { Rank (1-Highest } \\
\text { prevalence - Mpigi) }\end{array}$ & $\begin{array}{l}\text { Rank (1-Highest } \\
\text { prevalence - Gulu) }\end{array}$ \\
\hline \multirow[t]{10}{*}{ Cattle } & East coast fever & Amakebe & Oding ding & 1 & 1 \\
\hline & Coughing & Okukolola & Aona & 2 & 6 \\
\hline & Helminthosis & Enjoka & Kwidi, odini (liver flukes) & 3 & 2 \\
\hline & Heart water & Mulalama & $?$ & 4 & 9 \\
\hline & Mange & Olukuku & Angoli & 5 & 7 \\
\hline & Trypanosomosis & Kipumpuli & Tu o jonyo & 6 & 3 \\
\hline & Bovine ephemeral fever & Kamenyo & Okwero & 7 & 10 \\
\hline & Bloat & Kamukuulo & Deng ici & 8 & 5 \\
\hline & Ticks \& biting flies & Enkwa \& Ebiwuka & Okwodo \& Lwangu & 9 & 4 \\
\hline & Mastitis & Ebbani & Angoli me tunu & 10 & 8 \\
\hline \multirow[t]{5}{*}{ Goats and Sheep } & Helminthosis & Enjoka & Kwidi & 1 & 1 \\
\hline & Mange & Olukuku & Angoli & 2 & 3 \\
\hline & Heart water & Mulalama & Awila wic & 3 & 4 \\
\hline & Parapox or orf & Obumwamwa & Abworu & 4 & 2 \\
\hline & Abscesses & Ebizimba & Buu & 5 & 5 \\
\hline \multirow[t]{5}{*}{ Pigs } & African swine fever & Omusujja gw'embizzi & Orere pa opego & 1 & 1 \\
\hline & Helminthosis & Enjoka & Kwidi & 2 & 2 \\
\hline & Swollen udders & Okuzimba amabeere & Cak pa dyang/dyel ma kwot & 4 & 5 \\
\hline & Mange & Olukuku & Angoli & 3 & 4 \\
\hline & Lice & Ensekere & Nyugi & 5 & 3 \\
\hline \multirow[t]{5}{*}{ Chicken } & New castle & Kiwumpuli & Orere pa gwenu & 1 & 1 \\
\hline & Coccidiosis & Kiddukano & Orere & 2 & 2 \\
\hline & Helminthosis & Enjoka & Kwidi & 5 & 4 \\
\hline & Mites and fleas & Obuloolo, enkukunyi & Ladep, Lakuny & 3 & 5 \\
\hline & Flu & Senyiga & Abworo & 4 & 3 \\
\hline
\end{tabular}

difference between conventional and traditional drug use in Gulu and Mpigi districts $\left(x^{2}=24 ; \mathrm{p}<0.001\right)$. Farmers in Mpigi mentioned six plants (no Acholi names specified in Table 3), which were not used in Gulu. The latter mentioned one plant, Hyparrhenia rufa ("Ajuu") not used in

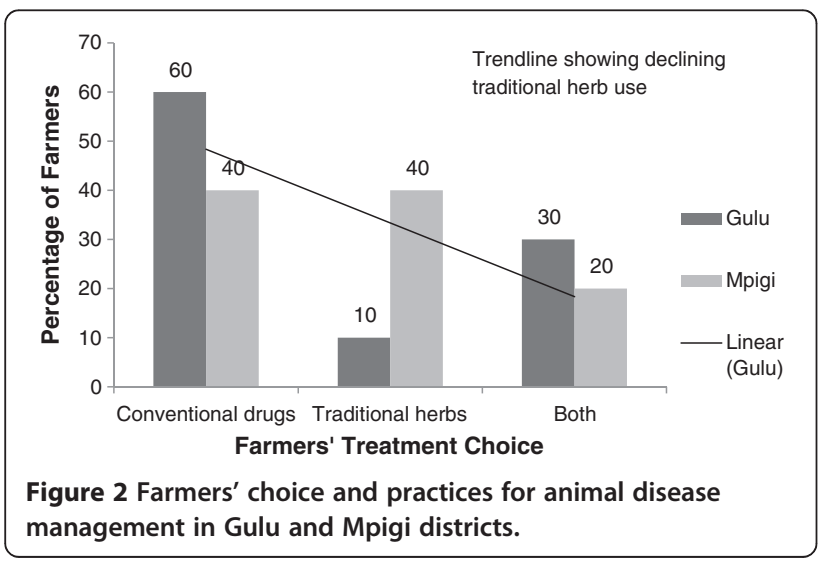

Mpigi. Different plant names in English, Luganda and Acholi were given (Table 3).

\section{Indigenous technical knowledge used}

This study established that 40 plants from 34 genera and 22 families are used to treat different diseases and conditions. Euphorbiaceae (15\%) followed by Solanaceae (13\%) were the commonest plant families. Twenty plants were used to treat helminthosis, wounds and ecto-parasites (8), theileriosis (6), retained placenta (5), bovine ephemeral fever (4), and one each for Newcastle disease, uterine prolapse, constipation and retained placenta. Some plants were indicated for more than one disease/condition. The resource persons (90\%) acquired the ITK through interand intra-generation oral tradition within the family. Most plants were used in fresh form. A few rare plants (10\%), tree leaves and roots were preserved in dried powder or whole form and constituted when needed. The oral route (80\%) for systemic conditions and topical application (15\%) for ecto-parasite control were the most common 
Table 3 Plants used to treat different diseases/conditions and how they are used

\begin{tabular}{|c|c|c|c|c|c|c|}
\hline $\begin{array}{l}\text { Relative availability*; } \\
\text { IUCN status }{ }^{\mathrm{a}}\end{array}$ & $\begin{array}{l}\text { Voucher } \\
\text { number }\end{array}$ & Family & Species and common name ${ }^{* * *}$ & $\begin{array}{l}\text { Vernacular name } \\
\text { (Luganda, Acholi) }\end{array}$ & $\begin{array}{l}\text { Habit; part } \\
\text { used**** }\end{array}$ & $\begin{array}{l}\text { Preparation method (Amounts vary with } \\
\text { species and age of animal) }\end{array}$ \\
\hline \multicolumn{7}{|c|}{ Disease/condition: Helminthosis in all livestock and poultry } \\
\hline $\begin{array}{l}\text { Common weed, } \\
\text { invasive: }+++; \mathrm{NE}\end{array}$ & NI 039 & Euphorbiaceae & $\begin{array}{l}\text { Euphorbia heterophylla Linn. Klotzsch \& } \\
\text { Garcke (Milk weed) }\end{array}$ & Kisandasanda & $H ; L$ & - Boil leaves and drench (too much causes diarrhoea) \\
\hline \multirow{2}{*}{$\begin{array}{l}\text { Rare weed by road } \\
\text { sides: +; NE }\end{array}$} & \multirow[t]{2}{*}{$\mathrm{NI} 002$} & \multirow[t]{2}{*}{ Caesalpinaceae } & \multirow{2}{*}{$\begin{array}{l}\text { Senna occidentalis (L.) Link } \\
\text { (Stinking weed) }\end{array}$} & \multirow[t]{2}{*}{ Muttanjoka; Ayila } & \multirow[t]{2}{*}{$S ; L, R$} & - Pound roots, add water and rock salt then drench \\
\hline & & & & & & - Crush fresh leaves, or use dried powder, add water and drench \\
\hline \multirow{3}{*}{$\begin{array}{l}\text { Ubiquitous, easily } \\
\text { located: }++ \text {; NE }\end{array}$} & \multirow[t]{3}{*}{$\mathrm{NI} 003$} & \multirow[t]{3}{*}{ Asteraceae } & \multirow{3}{*}{$\begin{array}{l}\text { Vernonia amygdalina Delile Kuntze }{ }^{a} \\
\text { (Bitter leaf) }\end{array}$} & \multirow[t]{3}{*}{ Mululuza; Labwori } & \multirow[t]{3}{*}{$S ; L, R, B$} & - Crush fresh leaves, add water and drench \\
\hline & & & & & & - Boil roots with water and give to drink \\
\hline & & & & & & - Give animal that has just delivered fresh leaves to eat \\
\hline \multirow{2}{*}{$\begin{array}{l}\text { Rare due to } \\
\text { restrictions: +; NE }\end{array}$} & \multirow[t]{2}{*}{ NI 036} & \multirow[t]{2}{*}{ Cannabaceae } & \multirow{2}{*}{$\begin{array}{l}\text { Cannabis sativa (Lam.) E. Small \& } \\
\text { Cronquist }^{b} \text { (Hemp, Marijuana) }\end{array}$} & \multirow[t]{2}{*}{ Njaga; Lakera } & \multirow[t]{2}{*}{$\mathrm{H} ; \mathrm{L}, \mathrm{S}$} & - Crush fresh leaves or add dried powder and mix with water \\
\hline & & & & & & - Crush, mix with water and crude lake salt and give to drink \\
\hline \multirow{2}{*}{$\begin{array}{l}\text { Wild, some grow it: } \\
++ \text { NE; APP } \|^{*}\end{array}$} & \multirow[t]{2}{*}{$\mathrm{NI} 038$} & \multirow[t]{2}{*}{ Xanthorrhoeaceae } & \multirow[t]{2}{*}{ Aloe vera (L.) Burm. $f$ (Babados aloe) } & \multirow[t]{2}{*}{ Kigaji } & \multirow[t]{2}{*}{$H ; L$} & - Slice and boil the leaves and give to drink \\
\hline & & & & & & - Slice fresh leaves and add to feeds \\
\hline \multirow{2}{*}{$\begin{array}{l}\text { Commonly grown for } \\
\text { sale: +++; NE }\end{array}$} & \multirow[t]{2}{*}{$\mathrm{NI} 021$} & \multirow[t]{2}{*}{ Solanaceae } & \multirow[t]{2}{*}{ Nicotiana tabacum L. (Tobacco) ${ }^{\text {c,d }}$} & \multirow[t]{2}{*}{ Taaba; Muvuavui } & \multirow[t]{2}{*}{$H ; L$} & - Crush leaves and mix with water then drench \\
\hline & & & & & & - Boil dried leaves, leave to cool and drench \\
\hline \multirow{2}{*}{$\begin{array}{l}\text { Commonly eaten in } \\
\text { households: }+++; \mathrm{NE}\end{array}$} & NI 040 & Solanaceae & Capsicum annuum (Dunal) & Kamulali; Obolo & $\mathrm{H} ; \mathrm{L}, \mathrm{Fr}$ & - Crush fruits or leaves, mix with ash and water then drench \\
\hline & & & Heiser \& Pickersgi & & & $\begin{array}{l}\text { - Crush fruits or leaves and mix with tobacco or cannabis and ash } \\
\text { then drench }\end{array}$ \\
\hline Common fruit: +++ ; & NI 037 & Caricaceae & Carica papaya L. Linn. (Pawpaw) & Papaali; Owak & $T ; R, S$ & - Roots crushed, boiled with water and little paraffin, then drench \\
\hline & & & & & & - Dried seeds crushed and boiled with water, then drench \\
\hline Ubiquitous: ++; NE & $\mathrm{NI} 005$ & Lamiaceae & $\begin{array}{l}\text { Tetradenia riparia (Hochst.) Codd } \\
\text { (Ginger-bush) }\end{array}$ & $\begin{array}{l}\text { Kyewamala; } \\
\text { Omwombyer }\end{array}$ & $S ; L$ & $\begin{array}{l}\text { - Crush leaves, mix with water and give to drink-Crush leaves and } \\
\text { put on the wound }\end{array}$ \\
\hline & & & & & & - Drench with decoction before calf suckles (ECF) \\
\hline $\begin{array}{l}\text { Ubiquitous by } \\
\text { roadsides: }+++ \text {; NE }\end{array}$ & $\mathrm{Nl} 001$ & Caesalpinaceae & $\begin{array}{l}\text { Senna hirsuta (L.) H. S. Irwin \& Barneby } \\
\text { (Stinking cassia) }\end{array}$ & $\begin{array}{l}\text { Gasia; Coga } \\
\text { macon }\end{array}$ & $S ; L$ & $\begin{array}{l}\text { - Boil dry leaves with little rock salt and drench or crush fresh } \\
\text { leaves and drench. }\end{array}$ \\
\hline & & & & & & - Cut and give the animals as fodder \\
\hline Wildly growing: $++;$ & $\mathrm{NI} 004$ & Leguminosae & Tephrosia vogelii Hook f. & Muluku; Kineke & $S ; L$ & - Crush fresh leaves and drench \\
\hline & & & (Fish-poison bean) & & & - Boil dried leaves, cool and drench \\
\hline Cultivated: ++; NE & $\mathrm{NI} 032$ & Euphorbiaceae & Jatropha curcas Linn. McVaugh & Kiloowa; Olwiro & $S ; L, F r$ & - Crush fresh leaves and fruits then drench \\
\hline & & & & & & - Crush leaves and put on the wound \\
\hline $\begin{array}{l}\text { Wildly growing: }++ \text {; } \\
\text { NE }\end{array}$ & $\mathrm{NI} 033$ & Euphorbiaceae & Ricinus communis Linn. (Castor oil) & Nsogasoga; Laliya & $S ; L, S$ & $\begin{array}{l}\text { - Decoction by boiling leaves and crushed seeds; cooling and } \\
\text { drenching }\end{array}$ \\
\hline
\end{tabular}


Table 3 Plants used to treat different diseases/conditions and how they are used (Continued)

\begin{tabular}{|c|c|c|c|c|c|c|}
\hline \multirow{2}{*}{$\begin{array}{l}\text { Wildy growing or } \\
\text { planted: }++; \text { NE }\end{array}$} & \multirow[t]{2}{*}{$\mathrm{NI} 006$} & \multirow[t]{2}{*}{ Meliaceae } & \multirow{2}{*}{$\begin{array}{l}\text { Azadirachta indica Linn. A. Juss. } \\
\text { (Neem tree) }\end{array}$} & \multirow{2}{*}{$\begin{array}{l}\text { Niimu; } \\
\text { Nyakanyaka }\end{array}$} & \multirow[t]{2}{*}{$\mathrm{T} ; \mathrm{L}$} & - Crush fresh leaves and drench \\
\hline & & & & & & - Crush fruits, boil them, cool and drench \\
\hline Rare herb: +; NE & NI 031 & $\begin{array}{l}\text { Leguminosae - } \\
\text { Papilionoideae }\end{array}$ & Pseudarthria confertiflora (A. Rich.) Baker & Kikakala & $H ; L, B$ & - Boil leaves or bark for one hour, cool and drench \\
\hline $\begin{array}{l}\text { Common weed, } \\
\text { invasive: }+++; \mathrm{NE}\end{array}$ & $\mathrm{NI} 027$ & Poaceae & $\begin{array}{l}\text { Digitaria abyssinica (A. Rich.) Stapf } \\
\text { (Couch grass) }\end{array}$ & $\begin{array}{l}\text { Lumbugu; Coga } \\
\text { macon }\end{array}$ & $G ; L, R$ & - Boil leaves and roots, cool then drench \\
\hline \multirow{2}{*}{$\begin{array}{l}\text { Wildly growing, rare: } \\
+; \mathrm{NE}\end{array}$} & \multirow[t]{2}{*}{$\mathrm{NI} 034$} & \multirow[t]{2}{*}{ Caesalpinaceae } & \multirow{2}{*}{$\begin{array}{l}\text { Senna didymobotrya (Fresen.) H. S. Irwin } \\
\& \text { Barneby (Peanut butter cassia) }\end{array}$} & \multirow{2}{*}{$\begin{array}{l}\text { Mukyula; Lakera/ } \\
\text { Lurogo }\end{array}$} & \multirow[t]{2}{*}{$\mathrm{T} ; \mathrm{L}$} & - Boil leaves, mixed with Senna occidentalis and drench \\
\hline & & & & & & $\begin{array}{l}\text { - Crush fresh leaves, mix with water and spray on skin } \\
\text { (ectoparasites) }\end{array}$ \\
\hline \multirow{2}{*}{$\begin{array}{l}\text { Grown in some } \\
\text { homes: ++; NE }\end{array}$} & \multirow[t]{2}{*}{$\mathrm{NI} 007$} & \multirow[t]{2}{*}{ Moringaceae } & \multirow[t]{2}{*}{ Moringa oleifera Lam. (Horseradish tree) } & \multirow[t]{2}{*}{ Moringa } & \multirow[t]{2}{*}{$\mathrm{S} ; \mathrm{L}, \mathrm{Fr}$} & - Crush fresh leaves, add ash and red pepper then drench \\
\hline & & & & & & - Boil dried leaves then cool and drench \\
\hline $\begin{array}{l}\text { Wildly growing: ++; } \\
\text { NE }\end{array}$ & NI 026 & Papilionoideae & $\begin{array}{l}\text { Erythrina abyssinica Lam. ex DC.e } \\
\text { (Flame tree) }\end{array}$ & Jirikiti; Lucoro & $\mathrm{T} ; \mathrm{B}$ & $\begin{array}{l}\text { - Pound the bark and leave to dry. Soak } 3 \text { handfuls of poun } \\
\text { dried bark in water ( } 2 \text { hours) and drench } 1 \text { cow or } 2 \text { goats }\end{array}$ \\
\hline \multirow{2}{*}{$\begin{array}{l}\text { Wildly growing: }++ \text {; } \\
\text { NE }\end{array}$} & $\mathrm{NI} 028$ & Phytolaccaceae & Phytolacca dodecandra L'Her. (Endod) & Luwoko; Olango & $\mathrm{H} ; \mathrm{L}, \mathrm{Fr}$ & - Infusion of the leaves, fruits then drench \\
\hline & & & \multicolumn{3}{|c|}{ Disease/ condition - African Swine Fever } & \\
\hline Practice 1 & & & \multicolumn{3}{|c|}{ Disease/ condition - New Castle Disease } & \\
\hline Practice 2 & & & & Human urine & N/A & - Mix with ash and rock salt, give to drink \\
\hline \multirow[t]{4}{*}{ In homes: ++; NE } & \multirow[t]{4}{*}{ NI 008} & \multirow[t]{4}{*}{ Solanaceae } & \multirow{3}{*}{$\begin{array}{l}\text { Capsicum frutescens L. Kuntze (Red } \\
\text { pepper) }\end{array}$} & \multirow[t]{3}{*}{ Kamulali; Pilipili } & \multirow[t]{3}{*}{$\mathrm{H} ; \mathrm{L}, \mathrm{Fr}$} & - Add ash + water to freshly squeezed leaves then drench \\
\hline & & & & & & $\begin{array}{l}\text { - Mix with Cannabis sativa, sisal (Agave sisalana) juice and as } \\
\text { drench }\end{array}$ \\
\hline & & & & & & - Mix with Aloe spp (Flower and leaf)+ Opium. \\
\hline & & & \multicolumn{3}{|c|}{ Disease/condition: Bovine Ephemeral Fever } & \\
\hline $\begin{array}{l}\text { Wildly growing: }++ \text {; } \\
\text { NE }\end{array}$ & NI 022 & Acanthaceae & $\begin{array}{l}\text { Acanthus pubescens (Thomson ex Oliv.) } \\
\text { Engl. (Grey goddess) }\end{array}$ & Amatovu; Achika & $S ; L$ & - Beat the legs with ends of the leaves until animal stands \\
\hline Wildly growing: + ; NE & NI 016 & Vitaceae & $\begin{array}{l}\text { Cyphostemma adenocaule Descoings. } \\
\text { ex Wild \& R. B. Drumm. (None specified) }\end{array}$ & Kibombo; Ogali & $H ; L$ & - Crush leaves, add ash and drench \\
\hline $\begin{array}{l}\text { In grazing lands: ++; } \\
\text { NE }\end{array}$ & NI 018 & Asteraceae & $\begin{array}{l}\text { Albizia anthelmintica Brongn } \\
\text { (Cherry-blossom tree) }\end{array}$ & $\begin{array}{l}\text { Mweramanyo; } \\
\text { Owak }\end{array}$ & $T ; L, B$ & - Decoction from leaves and bark then drench \\
\hline $\begin{array}{l}\text { Common weed: ++; } \\
\text { NE }\end{array}$ & NI 029 & Lamiaceae & $\begin{array}{l}\text { Leonotis nepetifolia (L.) R. Br. } \\
\text { (Sun-bird flower) }\end{array}$ & $\begin{array}{l}\text { Kifumufumu; } \\
\text { Okwero/ Achika }\end{array}$ & $\mathrm{H} ; \mathrm{L}$ & - Drench with warm decoction twice daily for three days \\
\hline Practice 3 & & & & & N/A & -Drench with decoction from boiled grass hoppers \\
\hline
\end{tabular}


Table 3 Plants used to treat different diseases/conditions and how they are used (Continued)

\begin{tabular}{|c|c|c|c|c|c|c|}
\hline \multicolumn{7}{|c|}{ Disease/condition: Constipation } \\
\hline Rare herb: +; NE & NI 035 & Cucurbitaceae & $\begin{array}{l}\text { Lagenaria sphaerica (Sond.) Naudin } \\
\text { (Wild calabash) }\end{array}$ & Kifuula; Lango & $H ; L$ & $\begin{array}{l}\text { - Drench of decoction made by boiling leaves, adding little rock } \\
\text { salt and cow ghee }\end{array}$ \\
\hline Practice 4 & & & & Cooking oil & N/A & Use large bore tube e.g. horse pipe to give orally \\
\hline Practice 5 & & & & $\begin{array}{l}\text { Omo liquid/very } \\
\text { soapy water }\end{array}$ & N/A & Give animal to drink \\
\hline \multicolumn{7}{|c|}{ Disease/condition: Theileriosis/ East Coast Fever } \\
\hline \multirow{2}{*}{$\begin{array}{l}\text { In grazing lands: +;NE, } \\
\text { APP } \|^{*}\end{array}$} & \multirow[t]{2}{*}{$\mathrm{Nl} 009$} & \multirow[t]{2}{*}{ Euphorbiaceae } & \multirow{2}{*}{$\begin{array}{l}\text { Euphorbia candelabrum Tremaux ex } \\
\text { Kotschy (Toothbrush tree) }\end{array}$} & \multirow{2}{*}{$\begin{array}{l}\text { Enkukuulu; Acak- } \\
\text { Acak }\end{array}$} & \multirow[t]{2}{*}{$\mathrm{T} ; \mathrm{La}$} & - Break and drop sap onto the lymph node or wound \\
\hline & & & & & & - Decoction drench removes afterbirth \\
\hline $\begin{array}{l}\text { Around paddocks: }++ \text {; } \\
\text { LC }\end{array}$ & $\mathrm{NI} 010$ & Euphorbiaceae & $\begin{array}{l}\text { Euphorbia tirucalli L. Klotzsch \& Garcke } \\
\text { (Pencil tree) }\end{array}$ & Lukoni, Labuka & S; La & - Break the leaves and put sap on the lymph nodes or wounds \\
\hline \multirow[t]{3}{*}{$\begin{array}{l}\text { Rare, in grazing lands: } \\
+; \mathrm{NE}\end{array}$} & \multirow[t]{3}{*}{$\mathrm{Nl} 025$} & \multirow[t]{3}{*}{ Lamiaceae } & \multirow[t]{3}{*}{$\begin{array}{l}\text { Clerodendrum myricoides (Hochst.) } \\
\text { Moldenke Butterfly weed }\end{array}$} & \multirow[t]{3}{*}{$\begin{array}{l}\text { Mukuzanyana; } \\
\text { Okwero }\end{array}$} & \multirow[t]{3}{*}{$S ; R$} & $\begin{array}{l}\text { - Pounded roots put in boiling water for one hour, cooled then } \\
\text { drench }\end{array}$ \\
\hline & & & & & & - Same to treat Bovine Ephemeral Fever \\
\hline & & & & & & - Same for helminthosis \\
\hline $\begin{array}{l}\text { Wildly growing: }++ \text {; } \\
\text { NE }\end{array}$ & $\mathrm{NI} 030$ & Asteraceae & $\begin{array}{l}\text { Microglossa pyrifolia (Lam.) Kuntze } \\
\text { (Secondary bush) }\end{array}$ & Akafugankande & $S ; L, R$ & $\begin{array}{l}\text { - Decoction of the roots then drench to relieve respiratory distress } \\
\text { in ECF }\end{array}$ \\
\hline Wildly growing: + ; NE & $\mathrm{NI} 012$ & Euphorbiaceae & $\begin{array}{l}\text { Euphorbia umbellata (Pax) Bruyns } \\
\text { (African milk bush) }\end{array}$ & Kafumba & $\mathrm{H} ; \mathrm{L}, \mathrm{FI}$ & - Break leaf and put sap on lymph node; irritate and dry the skin \\
\hline \multicolumn{7}{|c|}{ Condition - Lice, fleas, mites, mange and wounds } \\
\hline \multirow[t]{2}{*}{$\begin{array}{l}\text { Commonly grown: ++ } \\
+; \mathrm{NE}\end{array}$} & \multirow[t]{2}{*}{$\mathrm{NI} 020$} & \multirow[t]{2}{*}{ Poaceae } & \multirow[t]{2}{*}{$\begin{array}{l}\text { Sorghum bicolor (L.) Moench } \\
\text { (Sorghum) - Fermentation residue }\end{array}$} & \multirow[t]{2}{*}{$\begin{array}{l}\text { Muwemba; Any } \\
\text { wagi - Enkanja }\end{array}$} & \multirow[t]{2}{*}{ G; S } & $\begin{array}{l}\text { - Mix crushed fruits with roots of Cyphostemma adenocaule and } \\
\text { apply on wound }\end{array}$ \\
\hline & & & & & & - Smear on the skin \\
\hline $\begin{array}{l}\text { Wildly growing: ++; } \\
\text { NE }\end{array}$ & $\mathrm{NI} 023$ & Cucurbitaceae & $\begin{array}{l}\text { Momordica foetida Schumach. (Snake } \\
\text { food) }\end{array}$ & Bombo; Bomo & $H ; L$ & - Water extraction of the leaves, drench \\
\hline $\begin{array}{l}\text { Wildly growing: ++; } \\
\text { NE }\end{array}$ & \multirow[t]{3}{*}{ NI 015} & \multirow[t]{3}{*}{ Lamiaceae } & \multirow[t]{3}{*}{$\begin{array}{l}\text { Hoslundia opposita Vahl (White-tipped } \\
\text { hemizygia) }\end{array}$} & $\begin{array}{l}\text { Kamunye; } \\
\text { Odwongo }\end{array}$ & $S ; L, F I$ & $\begin{array}{l}\text { - Decoction applied daily externally with pressure on the wound } \\
\text { for a week or more }\end{array}$ \\
\hline Practice 6 & & & & Methylated Spirit & N/A & - Apply topically on wounds \\
\hline Practice 7 & & & & Soapy water & & - Add $O M O^{R}$ and wash the skin \\
\hline \multicolumn{7}{|c|}{ Disease/condition: Parturition failure } \\
\hline \multirow[t]{2}{*}{ Grown food: +++; NE } & \multirow[t]{2}{*}{$\mathrm{NI} 013$} & \multirow[t]{2}{*}{ Convolvulaceae } & \multirow{2}{*}{$\begin{array}{l}\text { Ipomoea batatas (L.) Lam (Sweet } \\
\text { potato) }\end{array}$} & \multirow[t]{2}{*}{ Amalagala; Maku } & \multirow[t]{2}{*}{$\mathrm{H} ; \mathrm{L}$} & - Give animal the vines to eat \\
\hline & & & & & & - Crush leaves without water and smear on the vulva \\
\hline
\end{tabular}


Table 3 Plants used to treat different diseases/conditions and how they are used (Continued)

\begin{tabular}{|c|c|c|c|c|c|c|}
\hline \multicolumn{7}{|c|}{ Disease/condition: Retained placenta } \\
\hline $\begin{array}{l}\text { Grazing land weed: } \\
++; \mathrm{NE}\end{array}$ & $\mathrm{NI} 014$ & Poaceae & $\begin{array}{l}\text { Hyparrhenia rufa (Nees) Stapf } \\
\text { (Jaragua grass) }\end{array}$ & Ajuu & $\mathrm{G} ; \mathrm{R}$ & $\begin{array}{l}\text { - Remove roots from stump, boil for one hour, cool and drench } \\
\text { animal }\end{array}$ \\
\hline $\begin{array}{l}\text { Wildly growing: }++ \text {; } \\
\text { NE }\end{array}$ & NI 019 & Anacardiaceae & $\begin{array}{l}\text { Rhus natalensis Bernh. ex Krauss } \\
\text { (Red cape beech) }\end{array}$ & $\begin{array}{l}\text { Kakwansokwanso; } \\
\text { Atakarach }\end{array}$ & $\mathrm{H} ; \mathrm{L} ; \mathrm{Fr}$ & $\begin{array}{l}\text { - Fresh fruits and leaves are crushed and mixed with water, sieved } \\
\text { and drenched }\end{array}$ \\
\hline Rare, in forests: +; NE & $\mathrm{NI} 024$ & Ebeneceae & $\begin{array}{l}\text { Diospyros abyssinica (Hiern) F. White } \\
\text { (African ebony) }\end{array}$ & Mpojwa & $\mathrm{T} ; \mathrm{L}, \mathrm{Fr}$ & - Leaves and dried fruits are crushed and a decoction is drenched \\
\hline \multirow{2}{*}{$\begin{array}{l}\text { Ubiquitous wildly } \\
\text { growing: }+++; \mathrm{NE}\end{array}$} & \multirow[t]{2}{*}{$\mathrm{NI} 011$} & \multirow[t]{2}{*}{ Solanaceae } & \multirow{2}{*}{$\begin{array}{l}\text { Solanum incanum (L.) Kuntze } \\
\text { (Sodom apple) }^{f}\end{array}$} & \multirow{2}{*}{$\begin{array}{l}\text { Entengotengo; } \\
\text { Ocuga }\end{array}$} & \multirow[t]{2}{*}{$\mathrm{H} ; \mathrm{L}, \mathrm{Fr}$} & - Smear crushed fresh fruit around the vulva \\
\hline & & & & & & - Squeeze sap from burnt fruit to wound \\
\hline \multirow[t]{2}{*}{$\begin{array}{l}\text { Wildly growing: }++; \\
\text { NE }\end{array}$} & \multirow[t]{2}{*}{ NI 017} & \multirow[t]{2}{*}{ Solanaceae } & \multirow[t]{2}{*}{$\begin{array}{l}\text { Solanum aculeastrum Dunal } \\
\text { (C. H. Wright) Bitter (Thorny apple) }\end{array}$} & \multirow[t]{2}{*}{ Kikutizangalabi } & \multirow[t]{2}{*}{$\mathrm{S} ; \mathrm{Fr}$} & $\begin{array}{l}\text { - Squeeze fruits in water or milk then given orally as drench } \\
\text { (cough) }\end{array}$ \\
\hline & & & & & & - Heat ripe fruits and smear on the vulva \\
\hline
\end{tabular}

*Availability of the plants varies; + Rare or endangered; ++ Available but not common; +++ Ubiquitous and quite common.

${ }^{* *}$ Month and year indicated for collection date.

${ }^{* * *}$ Month and year indicated for collection date.

****Habit: G: Grass; H: herb; Li: Liana; S: shrub; T: tree.

Plant parts: B - Bark; L - Leaves; La - Latex; Fl - Flowers; Fr - Fruits; R - Roots; S - Seeds.

Plant parts: $\mathrm{B}$ - Bark; $\mathrm{L}$ - Leaves; La - Latex; $\mathrm{F}$ - Flowers; $\mathrm{Fr}$ - Fruits; R - Roots; $\mathrm{S}$ - Seeds.
a Boil leaves or crush fresh leaves then drench; also used for stomach ache and fever in People or animals.

aBoil leaves or crush fresh leaves then drench; also used for stomach
bUsed on wounds, spray to prevent mites.

crush and mix whray to prevent mites.

cCrush and mix with water then spray animal to treat ectoparasites.

${ }^{d}$ Crush and boil leaves - drench treats uterine prolapse; decoction also treats cattle skin diseases including mange.

eAlso for estrus induction (bring animal to heat) and smear on the wounds for wound healing.

${ }^{\mathrm{f}}$ Also treats theileriosis and wounds.

Legend Table 3:

A summary of 40 plants and seven practices used in treatment of livestock and chicken diseases in Gulu and Mpigi districts. Details of preparation, plant part used, conservation/biodiversity status, common English, Acholi and Luganda names are provided. Twenty plants were used to treat helminthosis; wounds and ectoparasites (8), Theileriosis (6), retained placenta (5), Bovine Ephemeral Fever (4), and one each for Newcastle disease, uterine prolapse, constipation and retained placenta. 


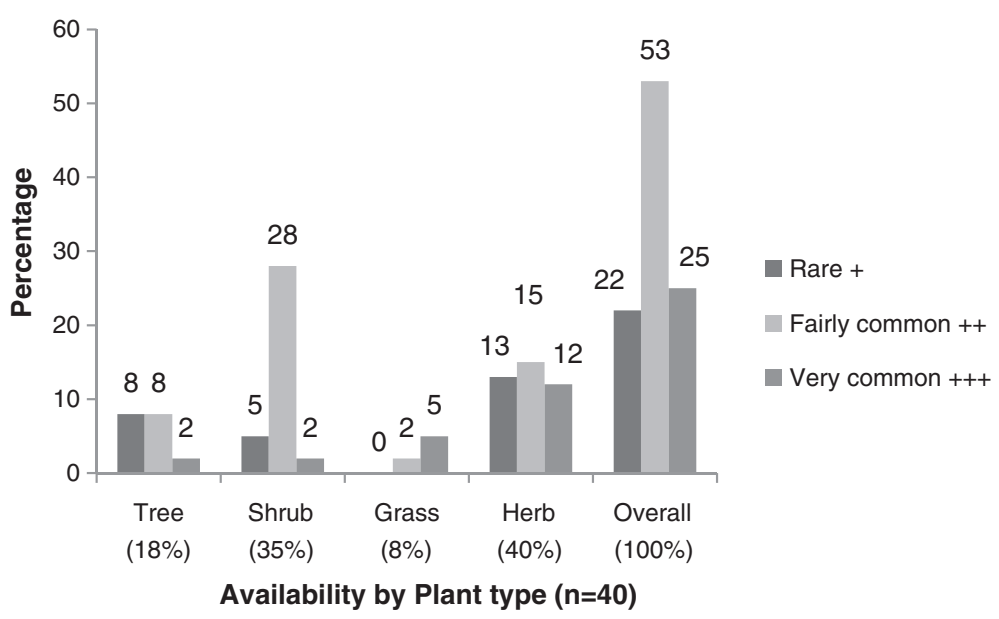

Figure 3 Relative availability of plant materials within communities.

modes of administration (Table 3). Leaves (75\%) bark (15\%) and roots $(5 \%)$ were most commonly used plant parts. Farmers knew the danger of relying on roots as the source of active components, as shown in this excerpt:

"We only use chips of roots from big trees so that we do not completely destroy the source. Most of these plants are rare and many people use the same tree; thus we do not encourage use of roots in traditional medicine", Female FGD Respondent, Gulu district.

Seven non-plant practices such as orally administering cooking oil and soapy water to treat constipation were cited. Human urine mixed with ash and a decoction of Ugandan edible grasshoppers were also used (Table 3).

\section{Perception of efficacy and risks of medicinal plants}

The majority (80\%) of farmers with knowledge of ITK said and believed the practices and plants were efficacious and safe, showing minimal side effects. Doses therefore were not standardized but indicated roughly. For example, a "mound" or "handful" of plant material mixed with a "bottleful" of water was more common expressions than quantifiable amounts or volumes. However, they said that some plants, such as Phytolacca dodecandra and Senna occidentalis, if used in large doses posed serious toxicological effects.

\section{Sources of herbal medicine}

Farmers obtained $>80 \%$ of the plants from wild flora or weeds, usually near their homes. About $15 \%$ of the shrubs or herbs were specifically grown or conserved around homes for medicinal purposes. The longest distance traveled for rare plant materials was $15 \mathrm{~km}$ in Gulu district. The majority (>70\%) of shrubs and forest trees were obtained within $2-6 \mathrm{~km}$. The access distances were shorter in Mpigi district and the longest distance was $5 \mathrm{~km}$ from designated forests. Herbs followed by shrubs were the most common plant types (Figure 3). A graph of relative availability of the plants demonstrated that $53 \%$ were fairly common; mostly the shrubs and $12 \%$ of the herbs were very common. Trees were rare or fairly common, depending on the species (Figure 3). International conservation status of $95 \%$ of plants was not evaluated (NE) by IUCN except Euphorbia tirucalli L. which was categorised as least concern (LC) and Carica papaya L. as vulnerable (VU). Categorisation by CITES indicated family Euphorbiacaea and Aloe vera as plants traded with caution (Table 3).

\section{Challenges}

Several challenges in using traditional knowledge and herbal medicine in treatment of livestock diseases were mentioned. The top three ranked were:

1. Most of the plants were used in combination, and limited access to some rare ones prohibited the full scale use of such ITK.

2. Most treatments were not openly shared, with few people having the knowledge.

3. The doses were not well standardized, with wide variations in treatment duration and amounts to administer.

\section{Discussion}

This is the first detailed survey of livestock ethnopharmacological practices from Gulu district. Evidently, there was lower utilization of ethnopharmacological practices in Gulu district compared to Mpigi. In Gulu district, younger farmers ( $<40$ years) portrayed less knowledge, possibly as a result of the breakdown in the 'traditional social safety nets' breakdown as an outcome of war or 
insurgencies in the district [36]. The role of traditional social safety nets adaptation and resilience has been recognized in Zimbabwe [37]. Similarly, goats instead of cattle were first priority due to reduced land sizes and cattle losses incurred during the previous war. The impact of the war is still felt in the economic performance of the district $[38,39]$.

The current study established 40 plants from 34 genera and 22 families, and 7 non-plant practices, used to treat livestock diseases. A previous global study documented that more than 119 conventional drugs prescribed were derived from plants. The bioactivity of $74 \%$ of 119 plantderived drugs was discovered during validation studies of documented traditional uses of the respective plants [40]. Therefore, the use of the plants identified in the present study in advanced searches for new drugs is paramount and can lead to novel discoveries. Documentation of ITK nevertheless, guards against loss of traditional knowledge due to limited inter-generation transfer [24,25].

More females than males participated, and a majority of the respondents at least had primary level education. Women have better health seeking behaviour than men, and herbal medicine is practiced in a similar pattern $[21,27]$. According to this study, men mainly did the ethno-diagnosis and treatment of livestock diseases. This is probably because taking care of large livestock such as cattle is by tradition considered male gender role in Uganda. This could also mean that much of ITK in livestock is mainly passed to the next generation through men. The participation of more women than men during FGDs could therefore mean that women considered this an opportunity to learn more about livestock. Indeed, FGDs in this study were a learning platform for many participants, who exchanged information on the different plants used for treating different diseases and conditions in livestock.

The numbers of plants and preparation methods described in this study were restricted to the prioritised diseases/conditions for the animal species identified (Table 2). In addition, the study documented the scientific, English, Luganda and Acholi names, which enhances intra- and inter-generation dissemination. The relative availability and conservation status of the different plants was reported, which few studies have achieved.

The most common mode of preparation was water extraction $(75 \%)$, where the plant parts were crushed and mixed with water before drenching. This is similar to previous findings [27] that showed the use of 37 plants against helminthosis in livestock from pastoral communities in Uganda. Decoction (boiling plant parts in water) was more common than infusion (submerging plant parts in hot water), and this is in agreement with a previous report [25]. However, this is contrary to a finding that most plant preparations in Bulamogi county, Uganda, were by infusion and less commonly by decoction [32]. The study in Bulamogi described more than 200 plants and practices for treatment of various human ailments but with less focus on livestock diseases.

Different studies have documented usage of some of these plant species in different parts of Uganda and Kenya [5,25,27,28,30,32,41,42]. All of these studies are area-specific, prospecting for different livestock or poultry diseases, whereas this study was focused on the farmer prioritized conditions and remedies. In one study, up to 29 plants were documented for the treatment of ECF, five for diarrhea and one against intestinal worms [32]. Another study [27] documented 11 of the plants mentioned in the current study but five of these were prepared differently. Such variations in methods may cause significant difference in bioactivity of plant material due to variation in concentration of bioactive compounds [3] even when the same plant is used. In a study of household herbal medicine used in four districts of Uganda, 41 plants were documented but noted the loss of ITK [31]. In pastoral Karamoja, 209 plants treating 130 conditions were documented [25]. A study in Mpigi district in 1993 documented 46 plants used for a wide range of conditions [5] but only $16(35 \%)$ of these were reported in the current study. What is interesting is the wide variation of ITK over time, even in the same or similar geographical locations.

Knowledge levels decreased by age, and the people younger than 40 years were less knowledgeable, which is similar to findings in Kenya [42]. Also similar to previous findings $[5,42]$, males in the present study were more knowledgeable than females of similar ages. The information was not freely shared, unlike in pastoral areas $[3,25]$, possibly because some people were believed to have native treating ability. The farmers believed most plants were safe and non-toxic [25,31]. However, plants known to be toxic were used, albeit with caution in advance. In these cases, there was an attempt to give clear amounts within which the doses were safe. Use of nonplant practices, like cooking oil and soap, has been documented before $[27,30,42]$. It is notable that these practices varied with the condition and usually no standard amounts were specified. The use of plants and non-plant practices in this study demonstrated that farmers have great wealth of knowledge of disease conditions affecting their animals. The validation of this information through well designed scientific research and dissemination of such findings will enhance ITK utilization.

\section{Conclusion and recommendations}

In Mpigi and Gulu districts, farmers used a variety of 40 plants and seven non-plant practices to treat livestock and poultry diseases. More than half of these plants were readily available in their environment while $25 \%$ were rare, and thus their use is compromised. The ethnoveterinary 
knowledge level was low among people below 40 years, especially in Gulu which may be attributed to the negative impact of prolonged war on the traditional social safety nets which are crucial in imparting ITK to the young folks. This was exhibited by fewer farmers practicing ITK in Gulu District. This calls for heightened sensitization about use and conservation of these plant resources in this district. Validation of efficacy of these ethnopharmacological products is also imperative to enhance full-scale use of these products in livestock production.

\section{Abbreviations}

ECF: East coast fever; FGDs: Focus group discussions; ITK: Indigenous technical knowledge; Klls: Key informant interviews.

\section{Competing interests}

The authors declare that they have no conflict of interest.

\section{Authors' contributions}

All authors contributed equally to this work. IN and KI took the lead in design and field implementation of the study, whereas JH, RA, and DO supervised the work and supported the writing process. JH, RA and IN contributed to data analysis and presentation. $\mathrm{RA}, \mathrm{KI}$ and $\mathrm{JH}$ supported the editing process. All authors read and approved the final manuscript.

\section{Acknowledgements}

This study was funded by the Swedish International Development Cooperation Agency (Sida) and Makerere University. We thank the farmers who allowed us to document this information about these plants and practices. The field data collection was supported by Ms. Sarifah Nabambejja, and Mr. James Ndukui helped us to preserve the plant materials for submission to the herbarium; we gratefully acknowledge their efforts. Mr. Mugagga Malimbo and Dr. Eva Tydén helped with drawing the study area map. Dr. Samuel Okech, Dr. David A. Morrison and Dr. Eva Tydén are thanked for editing this manuscript.

\section{Author details}

${ }^{1}$ College of Veterinary Medicine, Animal Resources and Biosecurity, Makerere University, P. O. Box 7062, Kampala, Uganda. ${ }^{2}$ Department of Biomedical Sciences and Veterinary Public Health, Section for Parasitology, Swedish University of Agricultural Sciences, P. O. Box 7063, Uppsala, Sweden. ${ }^{3}$ Department of Biosecurity, Ecosystems and Veterinary Public Health, P. O. Box 7062, Kampala, Uganda.

Received: 18 October 2013 Accepted: 18 December 2013 Published: 27 January 2014

\section{References}

1. Perry DB, Randolph TF: Improving assessment of the economic impact of parasitic diseases and their control in production animals. Vet Parasitol 1999, 84:145-168.

2. Lapenga KO, Rubaire-Akiiki C: The effect of helminthiasis on weight gains and carcass values of young indigenous goats in Uganda. J Anim Vet Adv 2009, 8(10):1993-1998.

3. Githiori JB, Hoglund J, Waller PJ: Ethnoveterinary plant preparations as livestock dewormers: practices, popular beliefs, pitfalls and prospects for the future. Anim Health Res Rev 2005, 6(1):91-103.

4. Rubaire-Akiiki C: Epidemiology of gastro-intestinal nematodes in cattle in dairy farms in Masaka district (Uganda), in parasitology and microbiology. Kampala: Makerere University; 1994:340.

5. Bizimenyera ES, Sebuguzi F, Kyewalabye E, Lubega GW: Ethnoveterinary practices in Mpigi district: use of medicinal plants in treating diseases of livestock. Tanzanian Veterinary Journal 2000, 20:16-26.

6. Ssewannyana E, Kabi F, Masembe C, Nabukenya I, Mwebembezi W: Current status of Brucellosis and helminthosis in cattle and goats in the southwestern rangeland zone of Uganda, In proceedings of the 3rd international e-conference on agricultural BioSciences. Kampala; 2010.
7. Cabaret J, Benoit M, Laignel G, Nicourt C: Current management of farms and internal parasites by conventional and organic meat sheep French farmers and acceptance of targeted selective treatments. Vet Parasitol 2009, 164(1):21-29.

8. Domke AVM, Chartier C, Gjerde B, Leine N, Vatn S, Osteras O, Stuen S: Worm control practice against gastro-intestinal parasites in Norwegian sheep and goat flocks. Acta Vet Scand 2011, 53.

9. Miller JE, Burke JM, Terrill TH, Kearney MT: A comparison of two integrated approaches of controlling nematode parasites in small ruminants. Vet Parasitol 2011, 178(3-4):300-310.

10. Chandrawathani P, Jamnah O, Waller PJ, Larsen M, Gillespie AT, Zahari WM: Biological control of nematode parasites of small ruminants in Malaysia using the nematophagous fungus Duddingtonia flagrans. Vet Parasitol 2003, 117(3):173-183.

11. de Araujo JV, Rodrigues MDD, Silva WW, Vieira LD: Biological control of gastrointestinal nematodes of goats in-semiarid climate by Monacrosporium thaumasium. Pesq Agrop Brasileira 2007, 42(8):1177-1181.

12. Nguyen TM, Van Binh D, Orskov ER: Effect of foliages containing condensed tannins and on gastrointestinal parasites. Anim Feed Sci Technol 2005, 121(1-2):77-87.

13. Minho AP, Gennari SM, do Amarante AFT, Abdall AL: Anthelmintic effects of condensed tannins on trichostrongylus colubriformis in experimentally infected sheep. Semina-Ciencias Agrarias 2010, 31(4):1009-1016.

14. Marie-Magdeleine $C$, Mahieu M, Philibert L, Despois $P$, Archimede H: Effect of cassava (Manihot esculenta) foliage on nutrition, parasite infection and growth of lambs. Small Rumin Res 2010, 93(1):10-18.

15. Sokerya S: The effects of cassava foliage (Manihot esculanta) on gastrointestinal parasites of small ruminants in Cambodia, In biomedical sciences and veterinary public health. Uppsala: Swedish University of Agricultural Sciences; 2009:75.

16. van Wyk JA, Hoste H, Kaplan RM, Besier RB: Targeted selective treatment for worm management-how do we sell rational programs to farmers? Vet Parasitol 2006, 139(4):336-346.

17. van Wyk JA, Bath GF: The FAMACHA ((c)) system for managing haemonchosis in sheep and goats by clinically identifying individual animals for treatment. Vet Res 2002, 33(5):509-529.

18. Coles GC, Jackson F, Pomroy WE, Prichard RK, von Samson-Himmelstjerna G, Silvestre A, Taylor MA, Vercruysse J: The detection of anthelmintic resistance in nematodes of veterinary importance. Vet Parasitol 2006, 136(3-4):167-185.

19. Sissay MM, Asefa A, Uggla A, Waller PJ: Anthelmintic resistance of nematode parasites of small ruminants in eastern Ethiopia: exploitation of refugia to restore anthelmintic efficacy. Vet Parasitol 2006, 135(3-4):337-346.

20. Hoglund J, Gustafsson K, Ljungstrom B-L, Engstrome A, Donnan A, Skuce P. Anthelmintic resistance in Swedish sheep flocks based on a comparison of the results from the faecal egg count reduction test and resistant allele frequencies of the b-tubulin gene. Vet Parasitol 2009, 161:60-68.

21. WHO: Decade of African traditional medicine 2001-2010, A serial publication of the WHO regional office for Africa. Geneva: The African Health Monitor; 2010.

22. Hammond JA, Fielding D, Bishop SC: Prospects for plant anthelmintics in tropical veterinary medicine. Veterinary Research Communication 1997, 21(3):213-228.

23. Grenier L: Working with indigenous knowledge: a guide for researchers, 1st ed. business \& economics, ed. IDRC: vol. 1. IDRC: IDRC; 1998:115.

24. Rao SVN, Van Den Ban AW, Rangnekar DV, Ranganathan K: Indigenous technical knowledge and livestock. In Handbook for straw feeding systems. Edited by Singh K, Schiere JB. New Delhi, India: ICAJR; 1995. http://www. edepot.wur.nl/21798

25. Grade JT, Tabuti JR, Van Damme P: Ethnoveterinary knowledge in pastoral Karamoja. Uganda. Journal of Ethnopharmacology 2009, 122(2):273-293.

26. Prichard RK, Geary TG: Drug discovery: fresh hope to can the worms. Nature 2008, 452(7184):157-158.

27. Nalule AS, Mbaria JM, Olila D, Kimenju JW: Ethnopharmacological practices in management of livestock helminthes by pastoral communities in the drylands of Uganda. Livest Res Rural Dev 2011, 23(2). http://www.lrrd.org/ Irrd23/2/nalu23036.htm.

28. Katunguka-Rwakishaya SK N, Sabiiti EN: Indigenous knowledge in ethno veterinary medicine in south western Uganda. DHP Publications series 
2004, 9. http://www.dspace.cigilibrary.org/jspui/bitstream/.../1/dhpindeginous-part-1.pdf.

29. Wasswa P, Olila D: The in vitro ascaricidal activity of selected indigenous medicinal plants used in ethno-veterinary practices in Uganda. Afr J Tradit Complement Altern Med 2006, 3:94-103.

30. Lagu C, Kayanja FIB: Medicinal plant extracts widely used in the control of Newcastle disease (NCD) and helminthosis among village chickens of South Western Uganda. Livest Res Rural Dev 2010, 22. http://www.Irrd.org/ Irrd22/11/lagu22200.htm.

31. Tabuti JRS: Catalogue of some household herbal medicines used in the districts of Nakapiripirit, Pallisa, Kanungu and Mukono. Kampala: S.M.K Dynamicprinters co. Ltd; 2011.

32. Tabuti JR, Dhillion SS, Lye KA: Ethnoveterinary medicines for cattle (Bos indicus) in Bulamogi county, Uganda: plant species and mode of use. J Ethnopharmacol 2003, 88(2-3):279-286.

33. Opiro R, Akol AM, Okello-Onen J: Ethnoveterinary botanicals used for tick control in the acholi subregion of Uganda. J Anim Vet Adv 2010, 9(23):2951-2954

34. UBOS: Uganda population and housing census, In main report. Kampala: The Uganda Bureau of Statistics; 2002.

35. Chambers R: The origins and practice of participatory rural appraisal, World development: vol. 22. Great Britain: Elsevier Science Ltd; 1994:953-969. http://www.alnap.org/pool/files/chambers-pra.pdf.

36. Isis-WICCE: Women's experiences of armed conflict in Uganda Gulu District, 1986-1999, Research report. Kampala: Isis-WICCE; 2000. http://www.isis.or.ug

37. Mapfumo P, Mtambanengwe F, Chikowo R: Mobilizing local safety nets for enhanced adaptive capacity to climate change and variability in Zimbabwe, NAPA (2007) climate change: Uganda national adaptation plan of action. Kampala: Ministry of Finance, Planning and Economic Development; 2010.

38. MFPED: Annual economic performance report 2010/11. Kampala: Ministry of Finance, Planning and Economic Development; 2011.

39. MAAIF: The National Livestock Census Report. Kampala: Ministry of Agriculture, Animal Industry and Fisheries, Uganda Bureau of Statistics (UBOS); 2008:273.

40. Farnsworth NR, Akerele O, Bingel AS, Soejarto DD, Guo Z: Medicinal plants in therapy. Bull World Health Organ 1985, 63(6):965-981.

41. Githiori JB, Hoglund J, Waller PJ, Leyden Baker R: Evaluation of anthelmintic properties of extracts from some plants used as livestock dewormers by pastoralist and smallholder farmers in Kenya against Heligmosomoides polygyrus infections in mice. Vet Parasitol 2003, 118(3-4):215-226.

42. Nanyingi MO, Mbaria JM, Lanyasunya AL, Wagate CG, Koros KB, Kaburia HF, Munenge RW, Ogara WO: Ethnopharmacological survey of Samburu district, Kenya. J Ethnobiol Ethnomed 2008, 4(14). http://www.ethnobiomed. com/content/4/1/14.

doi:10.1186/1746-4269-10-9

Cite this article as: Nabukenya et al: Ethnopharmacological practices by livestock farmers in Uganda: Survey experiences from Mpigi and Gulu districts. Journal of Ethnobiology and Ethnomedicine 2014 10:9.

\section{Submit your next manuscript to BioMed Central and take full advantage of:}

- Convenient online submission

- Thorough peer review

- No space constraints or color figure charges

- Immediate publication on acceptance

- Inclusion in PubMed, CAS, Scopus and Google Scholar

- Research which is freely available for redistribution

Submit your manuscript at www.biomedcentral.com/submit 